\title{
Nakagami Fading Impact on the Performances of VANET Routing Protocols in a Realistic Urban Area Setting
}

\author{
Hanene Brahmia \\ Networks and Systems Laboratory LRS, computer science department, \\ Faculty of Engineering Sciences, \\ Badji Mokhtar Annaba University, Algeria \\ Email: hanenebrahmia@gmail.com \\ Cherif Tolba \\ Networks and Systems Laboratory LRS, computer science department, \\ Faculty of Engineering Sciences, \\ Badji Mokhtar Annaba University, Algeria \\ Email: ctolba@yahoo.fr
}

ABSTRACT-

Quality of service is negatively impacted by the attenuation of the communication signal over the transmission distance. The attenuation phenomena can be modelled using different fading models; Nakagami model is regarded as the most realistic one. Attenuation in VANET is more challenging since it depends also on vehicle's length and node density. The main purpose of this paper is to evaluate the performances of Ad hoc On-demand Distance Vector, Dynamic destination-Sequenced Distance Vector and Optimized Link State Routing protocols. A real map from an urban zone has been used. The map has been prepared using the simulator of urban mobility (SUMO) for the network simulator 3 (ns-3). Results show that Ad hoc On-demand Distance Vector Routing Protocol outperforms the others being the most resistant to fading phenomena.

Keywords - AODV, DSDV, OLSR, VANET, Nakagami.

Date of Submission: Feb 12, 2020

Date of Acceptance: Feb 26, 2020

\section{INTRODUCTION}

V ehicular ad hoc networks (VANET) are nowadays an emerging technology that requires a robust communication infrastructure.

This technology is used in three major applications: [1]

- Safety: car diagnosis, route planning, emergency and warning messages.

- Traffic management: congestion, accident information, optimal speed warnings, smart parking.

- Comfort: instant messaging, multimedia streaming and downloading.

In our study, the traffic management and road safety are the major concerns, since the road accidents are increasing significantly. An intelligent transportation system should take place, to reduce the number of accidents, and make the traffic more fluid. Vehicular communication networks have been greatly developed aiming at sharing traffic information between road users that improves the efficiency of intelligent transportation systems [2] such as safety, comfort, pollution reduction and so on. Coordination of vehicles and dissemination of traffic information is vital in VANETs. Nevertheless, in some road traffic conditions, the delivery information could not ensure an early notification. For example, under congested traffic conditions, vehicles are forced to move at crawling speed. Hence, the wireless link quality in vehicle tovehicle $(\mathrm{V} 2 \mathrm{~V})$ communication can be affected due to Non Line-Of-Sight (NLOS) situations [3]. Furthermore, the broadcast storm problem can occur under higher node density, when several users attempt near simultaneous transmissions. Vehicular ad hoc networks are a sub class of mobile ad hoc networks (MANET) with some specificity like mobility criteria.

The mobility in MANET is not as challenging as in VANET, since the vehicles are moving in multiple areas: highway, rural, urban. Each area has its own configuration: number of lanes, directions, traffic units.

Several entities exist in these areas: pedestrians, roadside units, other types of vehicles (buses, cars, trucks), so there is a vehicle to everything V2X communication setting [4] [5]. In an intelligent transportation system, there are two main types of communication vehicle-to-vehicle (V2V) and vehicle to infrastructure (V2I). The routing challenges in VANET depend on the type of mobility, as the speeds of vehicles may interrupt the communication and cause link break. Routing performances degrade over distance because of the attenuation of the signal.

Signal propagation has been modelled using three types according to the authors of [6]:

1) Abstract models that do not reflect the reality

2) Deterministic models that depend on the distance between the sender and the receiver

3) Stochastic models that account for the non-deterministic effects caused by moving objects.

Nakagami is one of the deterministic models, which is applied in our study in addition to two-ray ground to evaluate the performances of VANET routing protocols. 
The main contribution of this article is the assessment of the influence of attenuation phenomena on the performances of the most used routing protocols. In order to select the better one for further use, because the choice of the protocol depends also on the traffic network topology and vehicular density. Using a real map trace from our city, and creating a realistic configuration with SUMO, then choosing the more realistic attenuation model. We choose three protocols from two classes: Ad hoc On-demand Distance vector AODV, from the reactive class. Dynamic destination-Sequenced Distance Vector DSDV and Optimized Link State Routing from the proactive class. Then, we compare packet delivery ratio (PDR), end-to-end delay and throughput for each protocol in different scenarios. The aim of vehicular ad hoc network simulations using network and mobility simulators is to create an artificial environment, which is as close as possible to real road networks where a real experimentation is not possible, expensive or risky. The paper is organised as follows: in section II we introduce VANET routing protocols, then in section III we present the mobility models used. Section IV is devoted to the propagation models. Some related works are discussed in section V. In section VI, simulation parameters are summarised. Results are presented and discussed in section VI. Finally, we conclude the paper in section VII with some perspectives.

\section{VANET Routing Protocols}

In an intelligent transportation system, a robust vehicle tovehicle communication is crucial. It needs reliable and efficient links to deliver the information correctly and timely. MANET routing protocols were called into question to verify their adaptability to a vehicular network. Routing protocols were categorised into five classes based on their routing strategy (fig. 1).

- 1. Topology: These types of protocols schematize the topology of the network in two manners:

On demand represented by the well-known ad hoc on demand distance vector protocol (AODV) or periodically like optimised link state routing protocol (OLSR) or a combination of the two techniques.

- 2. Location: Here the use of a localisation technique is crucial, in a delay or non-delay tolerance way.

- 3. Cluster: The network is divided into smaller cells. In each cell, a cluster head manages the communication and acts as a bridge. [7]

- 4. Broadcast: Traffic, area, cluster or probability broadcasting organises the routing depending on these configurations.

- 5. Geocast or multicast: The destination nodes are recognised by their geographical position.

AODV is known to be more suitable for frequent changing topologies since it is designed in a reactive way, which tolerates the neighbourhood changing. Its detailed presentation is described in [8].

We have also over-viewed his improvements to be more adequate to the vehicular networks in [9]. The main problem that degrades its performances is the density of vehicles since it's not a good scalable protocol. OLSR [10] is a proactive protocol that uses a neighbourhood table in which it stores all possible links to destinations. The unused paths in those tables consumes bandwidth what may degrades network performances.

DSDV uses AODV routing strategy based on distance vector and OLSR neighbourhood table-driven routing scheme with loop-free routing [11].

The three routing protocols are always on forefront for improvements and optimisation since they are standalone protocols that do not needs maps, global views and too much equipments. The motivation to make them more robust and efficient, is that in some cases extra information gathering is time consuming and costly.

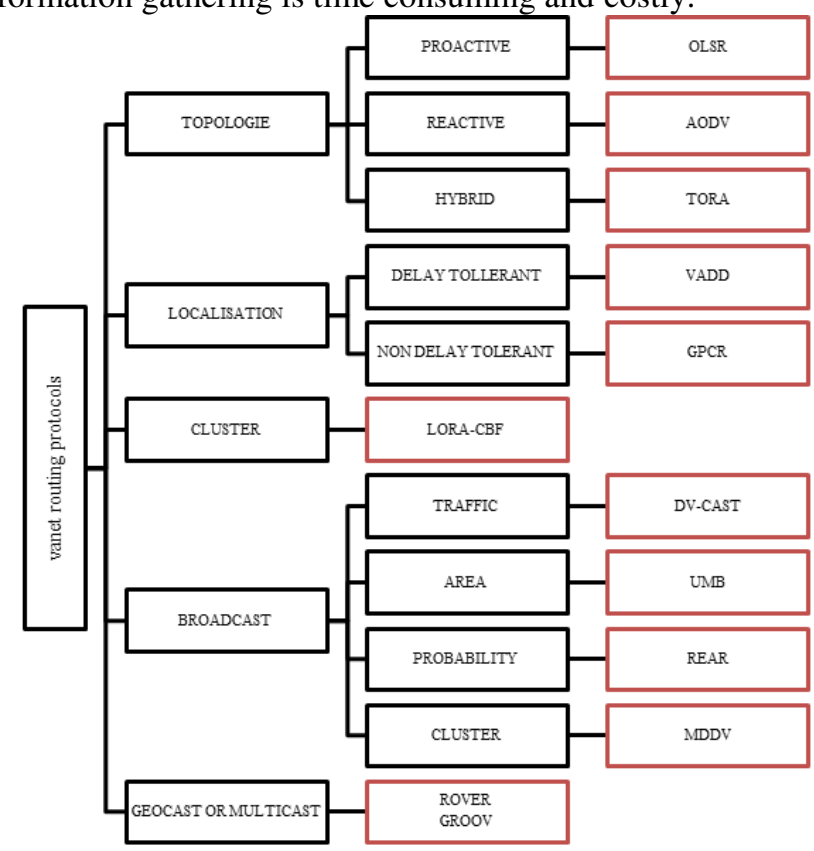

Figure 1 VANET routing protocols

\section{VANET MOBILITY MODELS:}

The major challenge in vehicular ad hoc networks resides in the mobility. Here, the direction of vehicles depends on road configuration and road side units. The speed is often limited. Although most simulations assume a random way point mobility, that don't reflect real situations. A realistic environment is preferred to test and evaluate the vehicle to vehicle and vehicle to infrastructure communication, so the combination of a mobility and network simulator is desired. The simulators may be isolated, embedded or federated. Vehicular mobility models are considered into five categories according to [12]:

- 1. Random: where the parameters like speed, heading and destination are sampled from a random process.

- 2. Flow: interactions between vehicles are modelled as flows.

- 3. Traffic: either trip using origin destination map or path defining end to end paths for individual cars or a flow of cars.

- 4. Behavioural: where there are no predefined rules but dynamic situation-adapted mobility. Artificial intelligence concepts can be used.

- 5. Trace based: using mobility traces generated by mobility simulators. 
Random way point mobility models were widely adopted due to their simplicity of implementation but then they were found inappropriate to vehicular networks due to their unrealistic behaviour. Despite that, a lot of research papers evaluated the performances of routing protocols with a random way point model.

In our study we are using trace mobility generated by the Simulator for Urban Mobility [13] software under NS3 to evaluate three most well adapted VANET routing protocols under fading situations. Movement traces give directly generic mobility patterns that reflect reality in a time saving manner.

Several mobility models were examined by the authors of [14] and Revival Mobility Model RMM was proposed. The proposed model gave good results in term of PDR, in a road of two or more lanes with randomly distributed nodes and different speed.

\section{Signal Propagation Models}

The network simulation idealizes somehow the vehicular environment. Many research papers have evaluated the performances of routing protocols without regard to the realistic aspects of signal propagation. As fading may cause communication failures, we use the Nakagami model to study the robustness of routing protocols, as it is able to implement different levels of fading.

Nakagami is one of the most realistic propagation models available. The authors of [6] classified and compared the propagation models as follows in (fig. 2).

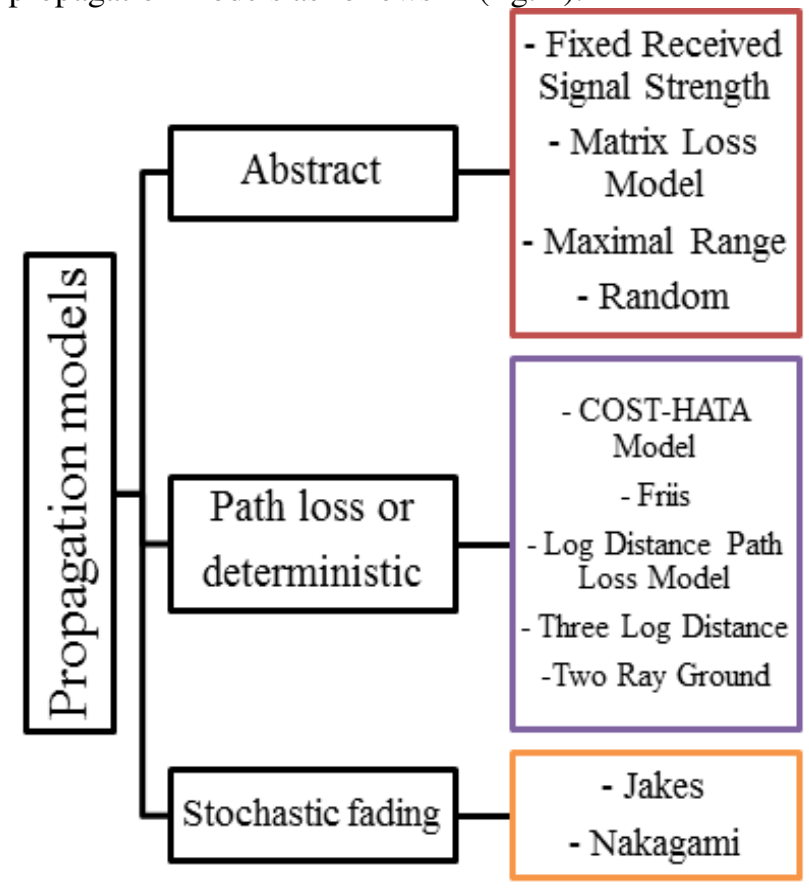

Figure 2 Propagation models

Abstract models need to be properly configured to model scenarios but often fail to model realistic ones. Some deterministic models predict path loss in urban areas, others use two rays: direct and ground reflected. Nakagami is based on a stochastic process which makes the simulation computationally expensive but more realistic. The probability density function is given by equation 1 :

$$
f(x ; \mu, \omega)=\frac{2 \mu^{\mu}}{\Gamma(\mu) \omega^{\mu}} x^{2 \mu-1} \exp ^{-\frac{\mu}{\omega} x^{2}}
$$

It is a stochastic model, where the signal power obeys a gamma $\Gamma$ distribution with two parameters omega $\omega=\left[\chi^{2}\right]$ the average fading power and mu $\mu>0$ the severity of fading, where the channel amplitude is $x \geq 0$. Here the signal reception power is determined using the probability distribution. The mathematical form of Nakagami is analytically tractable the reason why it is more suitable for fading modelling in wireless networks. Nakagami fading model matches empirical results for short wave ionospheric propagation.

It is relevant to model interferences from multiple sources. At the intersections or turns the distance between vehicles increases what causes changes in received signal strength. The average power changes also with distance. The goal of its use here in an urban environment is to overcome phenomena like distraction and interference caused by buildings and vehicles and to model interferences at intersections.

\section{RELATED WORKS}

The authors in [15] evaluated the performances of OLSR and AODV protocols under highly fading vehicular ad hoc network environment, they concluded that AODV outperformed OLSR in term of normalized routing overhead but failed in term of Packet delivery ratio and end to end delay. Changing hello and TC message intervals to lower values made from OLSR a better responsive protocol to network changes. Unfortunately the impact of the fading on the performances of the protocols has not been clearly identified. Fading impact on reactive protocols AODV and DYMO were evaluated by the authors of [16] using different propagation models. Evaluating the realistic models Ricean and Rayleigh beside free space and two ray ground with 50 nodes; varying the nodes' maximum speed and pause time conducted to degradation on the performances of the two routing protocols. But even using realistic propagation models maintaining node density cannot give better view that reflect the reality.

Random mobility also is to be avoided in a vehicular network. A radio propagation model was developed by [17] and compared with Nakagami and Two Ray Ground for more realistic simulations. DSDV, AODV and OLSR protocols were compared by each other in [18] using Nakagami propagation model, but with only seven nodes and generic simulation area. OLSR has been shown as the most optimum routing technique depending on throughput, packet loss ratio and packet overhead parameters. Nakagami and Log Normal models expressed a more accurate representation of the reality compared to TRG and Free Space in the work of [19]. They compared the four models on DYMO, OLSR, AODV and DSR protocols in urban VANET scenario. A new routing protocol has been developed by [20] considering Nakagami since it has been shown to well match the amplitude envelope of empirical data for Dedicated Short Range Communications (DSRC). A Manhattan grid was 
used by the authors of [21] to emulate vehicular mobility, in order to evaluate four routing protocols. Proactive protocols DSDV, OLSR and CLWPR outperformed AODV in an urban scenario where the channel fading was presented by Nakagami over other deterministic path loss models. The simulation was done for only 60 seconds and a limited density of vehicles with no support for CLWPR with wave in NS3. The authors of [22] integrated a routing metric that measures link stability taking into account multipath fading represented by Nakagami since it can model different fading environments in a flexible way. In a high way scenario they used eight road side units to connect vehicles to internet; Comparing three routing protocols LRP,LRP Enhanced and M-AODV+. The use of road side units does not help evaluating fading phenomena impact. Authors in [23] concluded that OLSR outperforms AODV and DSDV in term of PDR, goodput, routing overhead and end to end delay. A Manhattan grid was used in 100 seconds simulation scenario of 30, 40 and 50 nodes as maximum density. AODV, OLSR, DSDV, Dynamic Source Routing

DSR, Greedy Perimeter Stateless Routing GPSR, Zone

Routing Protocol ZRP and Cluster Based Routing Protocol CBRP were evaluated by [24] varying the shape factor of Nakagami in urban and highway scenarios concluded that there is not a single VANET protocol that suits all cases or scenarios. Researchers in [25] proposed a methodology for channel allocation to handle topology change based on the position of nodes in a cluster. The cluster head analyses bandwidth to efficiently share channel among nodes what improved overall network performance.

\section{Simulation PARAMETERS:}

Today's challenges in VANET simulation lie in generating traffic traces that are realistic and encompass various urban areas. To that end, different simulation tools are brought together to cover the different aspects of the vehicular area environment. We are taking an area from an urban zone of Annaba city in Algeria using Open Street Map (fig. 3) prepared using "Simulation of Urban MObility" (SUMO) (fig. 4) which is an open source, highly portable, microscopic road traffic simulation package, designed to handle large road networks. It has been extensively applied in different projects related to network performance, traffic assignment, vehicle routing, traffic impact analysis, traffic emission, V2X and other diverse traffic issues for more than 15 years. In our simulation, we have used SUMO beside the network simulator NS-3 to evaluate the performances of three routing protocols. We have then developed a script to derive packet delivery ratio, throughput and end to end delay. These evaluation metrics are measured for twenty four scenarios with varying parameters: vehicle density, routing protocol and fading.
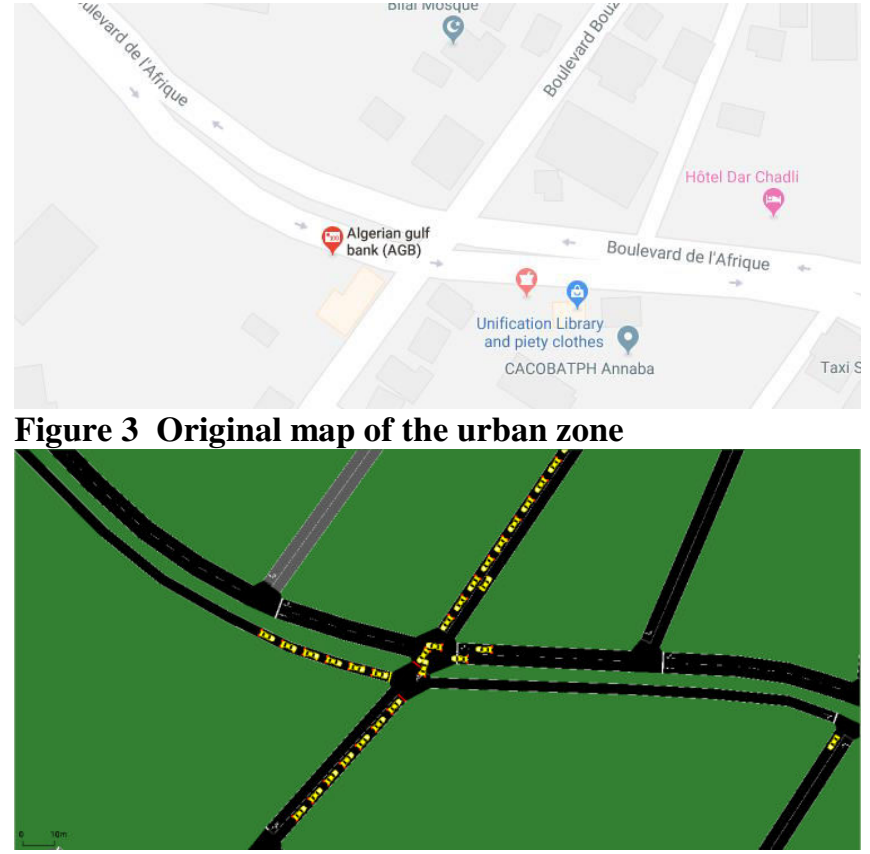

Figure 4 SUMO generated map

\section{RESULTS AND DISCUSSION:}

From (fig. 5) we can distinguish that DSDV was less affected by the fading phenomena than the other protocols in all node densities. At low densities DSDV had the lowest performance, while at 80 nodes it outperformed AODV. The density of vehicles at the intersection allowed a better delivery ratio for OLSR but not AODV. Overall scenarios OLSR outperformed the other protocols in no fading and fading environments.

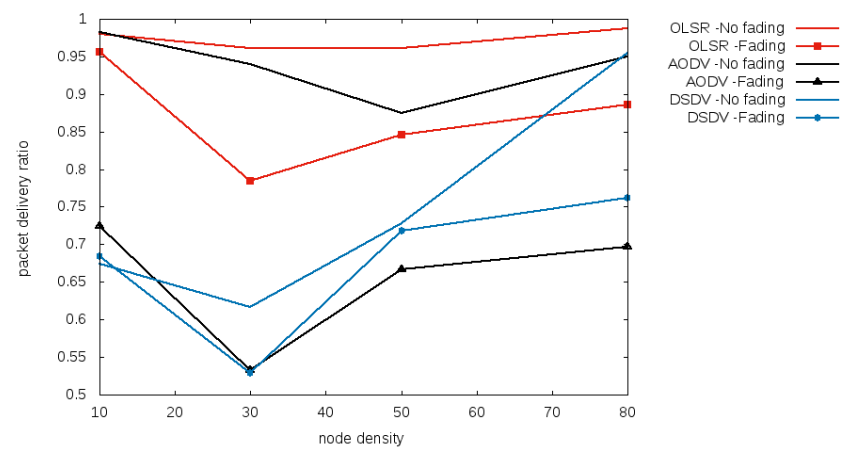

Figure 5 Packet Delivery Ratio.

When we compare the augmentation of end to end delay for the three protocols, we can distinguish that Adhoc Ondemand Distance Vector protocol outperformed the other protocols. The difference was significant only in the highest node density. At normal conditions OLSR had the lowest end to end delay, while DSDV and AODV had approximately same values. When we used Nakagami beside OLSR protocol the delay increased dramatically. In our scenario the two protocols using distance vector strategy outperformed OLSR as we can see in (fig. 6). 


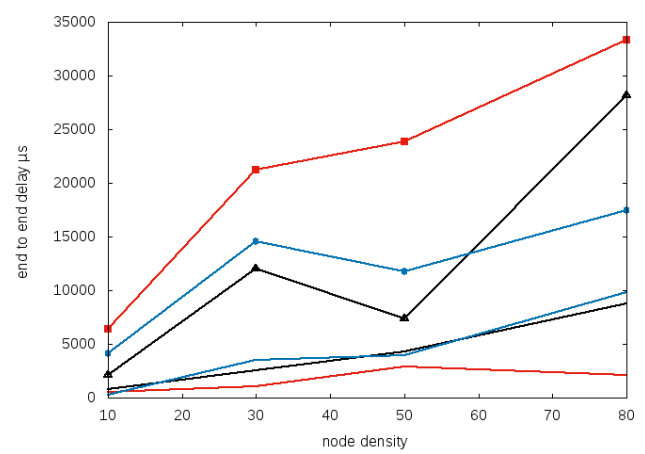

Figure 6 End to End delay

At 10 nodes the throughput for all routing protocols was alike; it had its best values. DSDV was the most influenced by the environment change while AODV outperformed the other protocols in the presence of fading effect. At higher node densities DSDV maintained good values for both scenarios dissimilar to AODV and OLSR. Overall we can see in (fig. 7) that AODV was better resistant under fading conditions in term of throughput.
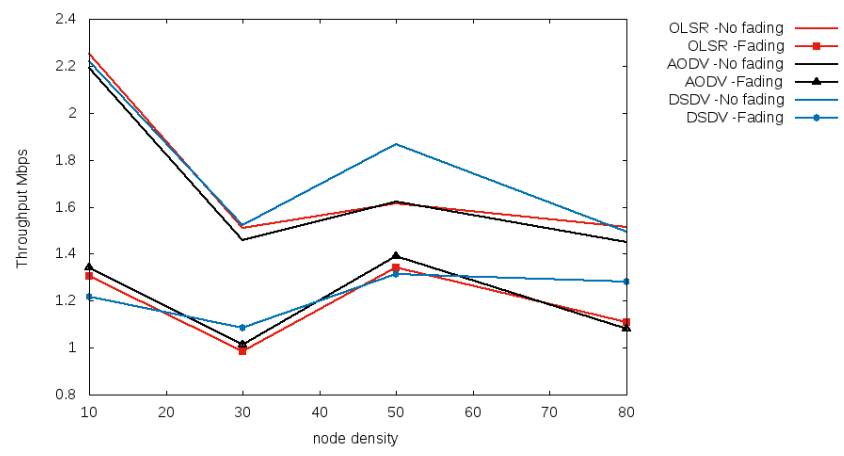

Figure 7 Throughput

From the three graphs presented in fig. 5, 6 and 7, we can conclude that in term of the best obtained values, OLSR protocol outperformed the other ones, but is the most influenced by fading phenomena. However in term of delay increase and throughput decrease we perceived that AODV is the best, but when node density gets higher its performances degrades. As mentioned before, DSDV is a proactive protocol from same class as OLSR that uses Distance Vector routing strategy as AODV. Although DSDV was always less robust than AODV, but better than OLSR under fading circumstances. Dissimilar to AODV, DSDV performed well in higher node densities.

\section{CONCLUSION}

In this paper, we have introduced the vehicular environment and its position inside the traditional network. We have summarised the VANET routing protocols and their challenges. Then we have chosen three routing protocols from two classes. These protocols are totally independent and do not need to collect huge amount of data to route the information. We studied the impact of fading on these protocols over an intersection scenario. Thus, we have evaluated their performances depending on three parameters: PDR, End to End delay and Throughput. According to our analyse of the simulation results, we distinguish that Ad hoc On demand Distance Vector protocol is more robust under fading. Although, it is not well scalable at higher node densities, while urban areas are denser compared to other road topologies. Giving AODV a global view of the network using Software Defined

Networks SDN for example, should improve its routing efficiency. This should reduce the delay caused by the route establishment process. The use of MANET adapted VANET protocols maintain a minimal service and were shown efficient. The dense networks and fast changing topologies make from establishing and maintaining routes a great challenge. The integration of other models and schematics is now more than necessary. And this will be our future work.

\section{REFERENCES}

[1] E.Schoch, F.Kargl, M.Weber and T.Leinmuller, 'Communication patterns in VANETs,' in IEEE Communications Magazine, 46(11), 2008, 119-125 [2] A.Ullah, X.Yao, S.Shaheen, and H.Ning, 'Advances in Position Based Routing Towards ITS Enabled FoG-Oriented VANET-A Survey', in IEEE Transactions on Intelligent Transportation Systems, 2003,1-13.

[3] O.Abumansoor, A.Boukerche, 'A Secure Cooperative Approach for Nonline-of-Sight Location Verification in VANET', in IEEE Transactions on Vehicular Technologie, 61(1), 2012.

[4] T.Wang, X.Wang, Z.Cui, Y.Cao and C.Suthaputchakun, 'Survey on cooperatively V2X downloading for intelligent transport systems', IET Intelligent Transport Systems, 13(1), 2019,13-21. [5] W.Tong, A.Hussain, W.X.Bo, and S.Maharjan, 'Artificial Intelligence for Vehicle-to-Everything: A Survey', IEEE Access, 7, 2019, 10823-10843. [6] M.Stoffers, and G.Riley, 'Comparing the ns3 Propagation Models', IEEE 20th International Symposium on Modeling, Analysis and Simulation of Computer and Telecommunication Systems, Washington, DC. 2012, 61-67.

[7] B. Ramakrishnan, R. S. Rajesh, S. Shaji, 'CBVANET: A Cluster Based Vehicular Adhoc Network Model for Simple Highway Communication', Int. J. Advanced Networking and Applications, 02(04), 2011, 755-761.

[8] C.Perkins, E.Belding-Royer, S.Das, 'Ad hoc On-Demand Distance Vector (AODV) Routing', RFC 3561, 2003.

[9] H.Brahmia, C.Tolba, 'Vanet routing protocols: discussion of various Ad-hoc On-demand Distance Vector (AODV) improvements'. Proc. of the The $3^{\text {rd }}$ International Conference on Pattern Analysis and Intelligent Systems, 2018, 276-281.

[10] T.H.Clausen, and P.Jacquet,'Optimized Link State Routing (OLSR)', RFC 3626, 2003.

[11] C.E.Perkins, and P.Bhagwat, 'Highly dynamic destination-sequenced distance vector routing (DSDV) for mobile computers' in Proc. ACM SIGCOMM 94, London, $U K, 1994,234-244$. 
[12] H. Hartenstein, K. Laberteaux, 'VANET: Vehicular Applications and Inter-Networking Technologies',109$139,2010$.

[13]https://www.dlr.de/ts/en/desktopdefault.aspx/tabid$1213 /$

[14] K.Prasanth, Dr.K.Duraiswamy, K.Jayasudha, Dr.C.Chandrasekar, "Packet Transmission Analysis in Vehicular Ad Hoc Networks using Revival Mobility Model," Int. J. Advanced Networking and Applications, 01 (01) 2010,252-257.

[15] I.Khan, and A.Qayyum,'Performance Evaluation of AODV and OLSR in Highly Fading Vehicular Ad hoc Network Environments' IEEE $13^{\text {th }}$ International Multitopic Conference, Islamabad, 2009, 1-5. [16] L.Nassef, 'on the effects of fading and mobility in on-demand routing protocols' Egyptian Informatics Journal, 11, 2010, 6774.

[17] F.J.Martinez, M.Fogue, M.Coll, J.-C. Cano., C. T Calafate., P.Manzoni 'Assessing the Impact of a Realistic Radio Propagation Model on VANET Scenarios using Real Maps' Ninth IEEE International Symposium on Network Computing and Applications, 2010, 132-139. [18] T.S.Chouhan, R.S.Deshmukh, 'Analysis of DSDV,OLSR and AODV Routing Protocols in VANETS Scenario: Using NS3' International Conference on Computational Intelligence and Communication Networks, 2015, 85-89.

[19] W. Angeles, V.P. Borin, A. Munaretto and M. Fonseca 'The Impact of Propagation Models in the Performance of Ad Hoc Routing Protocols for Urban VANET' IEEE 84th Vehicular Technology Conference (VTC-Fall), Montreal, QC, 2016, 1-5.

[20] W.Wang, T.Luo,'The Minimum Delay Relay Optimization Based on Nakagami Distribution for Safety Message Broadcasting in Urban VANET' in IEEE Wireless Communications and Networking Conference (WCNC 2016) - Track 3 - Mobile and Wireless Networks, 2016, 1-6.

[21] H.Sharm, T.Jayavignesh,'Comparative analysis and applicability of routing protocols in VANETs,' in International Conference on Electrical, Electronics, Communication, Computer, and Optimization Techniques (ICEECCOT), Mysuru, 2017, 1-8.

[22] D.Abada, A.Massaq, and A.Boulouz,'Connecting VANETs to Internet over IEEE 80211p in a Nakagami fading channel,' in International Conference on Wireless Technologies, Embedded and Intelligent Systems (WITS), Fez, 2017, 1-6.

[23] E.E.Akkari Sallum, G.dos Santos, M.Alves and M. M.Santos, 'Performance analysis and comparison of the DSDV, AODV and OLSR routing protocols under VANETs,' in 16th International Conference on Intelligent Transportation Systems Telecommunications (ITST), Lisboa, 2018, 1-7.

[24] B.Barmada, N.Alobaidi, I.Ardekani, M.Erfanian. Sabaee, and G. Ramirez-Prado,'Evaluating VANET Routing Protocols for Auckland Area.' in Eleventh International Conference on Mobile Computing and Ubiquitous Network (ICMU), Auckland, 2018, pp. 1.
[25] M.Abhilash Kumar Reddy, A. Rama Mohan Reddy, 'PBMAC-Position based channel allocation for vehicular ad hoc networks' International Journal of Advanced Networking and Applications, 8(4),2017, 3156. 